\title{
HEp-2 fluorescence pattern classification
}

\author{
V. Snell, W. Christmas, J. Kittler \\ CVSSP, University of Surrey, Guildford, UK \\ v.snell@surrey.ac.uk
}

\section{Introduction}

This work is aimed at improving the performance of automated diagnosis of auto-immune diseases from indirect immunofluorescence (IIF) images. A wide variety of these diseases affect different parts of the body, but are all associated with an immune reaction to, and an attack on, the person's own tissues. This reaction, known as Anti-nuclear antibody (ANA), can be visualised using indirect immunofluorescence, most commonly utilising the HEp-2 cell line, and forms the most reliable basis for ascertaining the presence of, and establishing the specific type of auto-immune disease. The diagnosis is usually performed by highly trained physicians directly at the microscope, although better results can be obtained through digital imaging of the slides, as the fluorescence decays fairly rapidly. Both the overall brightness and the visual pattern of the fluorescence feed into the diagnostic decision, although many clinical settings will only use the brighter samples, known as positive, for identification of specific patterns.

A large number of these visual patterns of fluorescence is described in the medical literature, and various groups or subsets of these have been targeted for automatic recognition by previous published works in the computer vision field. The HEp-2 Cells Classification contest [1] at ICPR 2012 recognised the difficulty in comparing these works, and provided a single dataset for "the comparison of systems able to automatically recognize the pattern of cells within IIF images [... on a large and significant set of real data", as well as broadening the interest in this application area. We describe the images from the contest dataset, and the classes they represent, in the following section; analysis of previous works and contest outcomes is given in Section 1.2, followed by an outline of our alternative approach in Section 1.3.

\subsection{Subject images and classes}

The data consists of IIF images of individual cells, each having an associated binary mask (removing issues of segmentation from any comparison), an intensity label (positive or intermediate), and a ground-truth class label from one of 6 classes. The classes are as follows:

- Homogeneous: a diffuse pattern, fairly uniform across the whole nucleus. 
- Fine speckled: a very fine-grained isotropic texture, not dissimilar to white noise.

- Coarse speckled: an isotropic texture of somewhat larger specks.

- Centromere: this class is characterised by large numbers of strong bright spots on a darker background. These are 2-3 pixels across, and 40-60 are supposed to be present, although in a number of intermediate intensity examples of this class none are visible to the eye, even after contrast normalisation.

- Nucleolar: a small number (less than 6) of larger bright areas within the nucleus.

- Cytoplasmatic: these nuclei are characterised by a strongly irregular shape, as compared to the generally elliptic nature of all other classes. The texture is equally irregular.

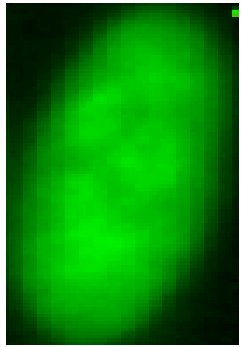

(a) Homogeneous

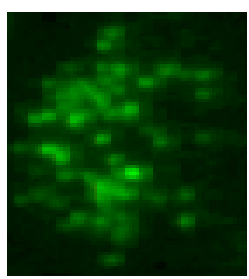

(d) Centromere

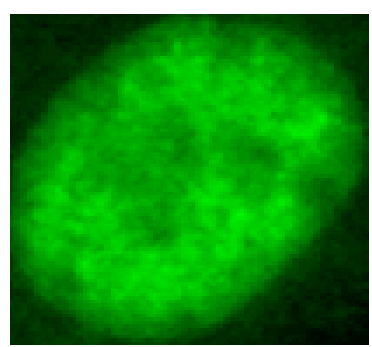

(b) Fine speckled

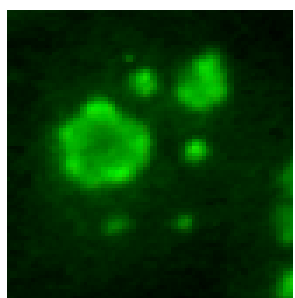

(e) Nucleolar

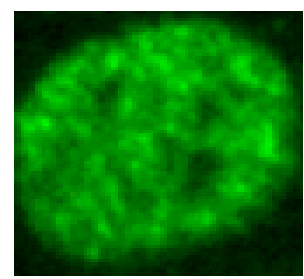

(c) Coarse speckled

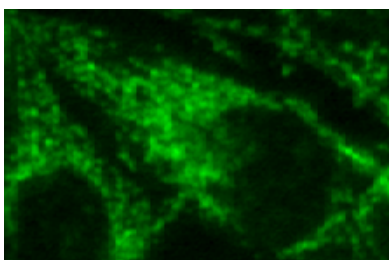

(f) Cytoplasmatic

Figure 1: Positive examples of each class

Examples of each class are given in Fig. 1, contrast boosted to make their features visible in print. Typical contrast range for positive examples is around 120 grey-levels, but can be as low as 25 levels for cells in intermediate samples, greatly exacerbating the effect of sensor noise. Image sizes range from 45 to 130 pixels across.

The images suffer from a number of artefacts: sensor impulse noise affecting groups of between 4 and 8 pixels across appears in a number of locations; 4pixel wide vertical banding is visible in areas of high gradients, and probably originates from a crude up-sampling algorithm in the scanning device. Finally, a variation in focus precision, which can affect textural measurements, is present 
within the image set.

\subsection{Previous works}

Computer vision researchers have attempted to automate classification of ANA IIF patterns for several years now. Although it is difficult to compare their results directly, as they use different private data sets and variable class definitions, the error rates for identification of individual cell patterns range around $10-25 \%[2,3,4]$. This is a promising start for early works, but not sufficient for widespread clinical application.

A public data set would provide a consistent base-line for comparing potential approaches to solving this problem. The HEp-2 Cells Classification contest [1], held at ICPR 2012, supplied such a base-line and attracted the participation of 28 groups from across the world, evaluating a rich variety of algorithms. The contest data set was split into training and withheld test portions, with 2-3 patient samples of each class in each portion. Class labels, which were assigned by the expert pathologist to the entire sample, were applied to all cells originating from that sample. Cells from all the samples within the training set were mixed up, with no annotation as to which cell came from which sample. Therefore any possible cross-validation arrangement which could be used for algorithm selection or parameter tuning by the contestants would necessarily include cells from the same sample in both training and validation sections. The performance estimates provided by such cross-validation (error rates of around $5 \%$ were reported by a number of participants) proved very far from the final test-set performance reported in [5], as the validation task is very much easier than the hold-out test, which contains genuinely independent samples from patients not represented in the training set.

The best cell-level accuracy achieved in the contest was just short of $70 \%$, which is comparable to earlier works, and also close to performance obtained by an expert pathologist operating under the same conditions as the contest algorithms, i.e. examining each cell individually, without the context of the rest of the sample. A major reason for the pathologist's difficulty is that for some classes, the pattern definition actually relies on the exact appearance of relatively rare mitotic cell(s) which were present in the slide, but not included in the contest data set. Appearance of these classes is otherwise very similar to each other, and they generate a large proportion of the confusions.

The range of features employed by the participants, which are summarised in [5] and covered in more detail in a number of papers published at ICPR ([6, $7,8,9,10,11,12,13])$, is quite broad. Most algorithms included some form of texture measurement, and often a combination of several; co-occurrence matrices, Local Binary Patterns, and various extensions thereof, as well as gradients, frequency transforms and Gabor wavelets. Morphological and granulometry features form another strand, recognised by several researchers as relevant to these image patterns. Convolutional, dictionary learning, randomized and evolutionary feature extraction methods were also represented. As a very broad trend within the contest results, methods which employed a large number of features (many hundreds), in combination with a linear classifier, generalised somewhat 
better under these conditions than algorithms with a smaller feature set and more sophisticated non-linear classifiers.

\subsection{Overview}

In this paper, we highlight the limitations of existing approaches to HEp-2 pattern classification, by performing comparative experiments on a number of different feature sets and assessing the degree of connection between cell-level accuracy and predictive capacity for whole-sample decisions. We attempt to overcome these limitations by proposing the use of a distance metric for sets of cells, which can take into account the full set of measurements from a patient's sample, rather than narrowing the cell information down to a hard class decision before allowing it to be combined with information from other cells within the sample. We show that this approach has a stronger connection to the ultimate goal of performing a clinical diagnosis, and provides the researcher with a richer insight into the causes of confusion.

We describe the different protocols that are used to compare features in Section 2.1, and full details of the feature sets themselves are given in Section 2.3. Experimental results for each combination of feature set and evaluation protocol are listed in Section 3 and their implications are analysed in Section 4.

\section{Methods}

This section describes the experimental protocols employed to obtain the results, data sets used, and a number of feature sets that are compared for their suitability. As there is no suitable base-line benchmark to compare against, we evaluate several different descriptors to compare against each other, and assess the variability of results. We constrain ourselves to the classic pipeline of a fixed feature extraction step followed by a classifier, and use multi-class SVM with RBF kernel, whose hyper-parameters are determined by a grid search.

\subsection{Experimental protocols}

Three experimental protocols are reported in this paper: the original contest protocol, a sample-based cross-validation procedure with majority vote by individual cells, and a method which considers the distribution of cell parameters within a sample.

\subsubsection{Contest protocol}

The original contest data set was split into training and withheld test portions, with separate patient samples used for each portion. The training set contained 2-3 labelled samples from each class, but cells from all the samples were mixed together, with no information on which cell came from which sample. Such a data set only allows for cell-based cross-validation within the training portion, resulting in folds which contain cells from the same sample in both training and validation sections. Under these conditions, methods that are sensitive to a sample's specific imaging characteristics (such as focus or contrast), 
rather than broader class characteristics, can provide a very accurate prediction for the validation set, but may not generalise well to the held-out test. For this protocol, we are requested to report both the cell-level confusion matrix for the test set, and the sample-level confusion, obtained by a majority vote of cells within each sample. We compare these to the average accuracy obtained by cross-validation within the training set in order to assess the generalisation performance.

\subsubsection{Sample-based cross-validation}

The protocol recommended by the contest organisers for further research addresses the problems of the contest protocol by using additional information about the source sample for each cell. It is a leave-one-out procedure, where all cells from a single sample are held out as validation set for each fold. This gives a much fairer assessment of the expected real-life performance of a classification method, and has the additional benefit of a much larger training set, with 4-6 independent samples of each class available for training in each fold. Prediction of class label is still made independently for each cell, without making use of any information from other cells within the same sample.

\subsubsection{Cell distribution}

Human experts assessing a sample take account of the appearance of all its cells together. To reflect this, we additionally report the results of estimating the distribution of cell parameters within each sample, and compare distribution overlap for samples of the same class and from different classes. A feature extraction process which is invariant to the 'distractor' variables, such as differences in overall sample intensity or focus, while being sensitive to the true class-dependent characteristics of the image, should produce distributions that overlap strongly with those from the same class, while being well separated from distributions of other classes. We use the Bhattacharyya distance (denoted $D_{B}$ ) for multivariate Normal distributions, based on sample mean and full co-variance, calculated according to Eq. 1, where $\mu_{1}$ and $\mu_{2}$ are sample means of the two distributions, $\Sigma_{1}$ and $\Sigma_{2}$ are the corresponding co-variances, and $\Sigma=\left(\Sigma_{1}+\Sigma_{2}\right) / 2$.

$$
D_{B}=\frac{1}{8}\left(\mu_{1}-\mu_{2}\right) \Sigma^{-1}\left(\mu_{1}-\mu_{2}\right)^{T}+\frac{1}{2} \log \left(\frac{|\Sigma|}{\sqrt{\left|\Sigma_{1}\right|\left|\Sigma_{2}\right|}}\right)
$$

The normality assumption holds better for some feature sets than for others. We produce distance maps for a variety of feature sets to illustrate their strengths and weaknesses.

\subsection{Data sets}

The contest training data consists of 721 images of individual cell IIF patterns, with a further 734 cell images in the test portion. The classes are approximately, though not precisely, balanced, and roughly equal numbers of positive and intermediate examples of each class are included. 
An expanded dataset [14], released by MIVIA after the completion of the contest, additionally contains the whole field images captured by the microscope, and binary masks identifying the location of each cell within the sample. This allows for sample-based evaluation, and is used for both majority-vote and distribution-based protocols. There are 28 samples altogether, each is 1388 pixels wide by 1038 high, with number of cells per sample varying between 13 and 119 .

\subsection{Feature sets}

As the class descriptions in Section 1.1 make clear, most of the distinctions between HEp-2 patterns are based on textures. With this in mind, we compare a number of different approaches to texture measurement against each other. As the cytoplasmatic class is also characterised by shape, we include circularity of the mask (calculated as area divided by square of the perimeter) as shape descriptor in every feature set. All feature vectors also include the basic measurements of pixel value average within the cell mask, and their contrastnormalised standard deviation. We also note that all the textures are completely isotropic, allowing simplified formulations compared to the general case. As the fluorescence is monochromatic, we further simplify texture assessment by only using the dominant green component of the images.

\subsubsection{DCT based descriptor}

We note from the class descriptions that their distinctions are often ones of scale, rather than a specific textural pattern. This is most apparent in fine vs. coarse speckled cases, but also continues to larger spots in centromere, and even larger bright areas in nucleolar. We therefore use the power spectrum to capture the scale at which textural variation is strongest, as described in greater detail in [10].

The frequency analysis is performed as a 32-point DCT of line sections from inside the segmented mask boundaries. As the texture is isotropic, a 1dimensional transform is sufficient to establish its frequency distribution. Transforms from all the qualifying lines within a cell image are averaged to reduce variability and noise, and intensity normalised by min-to-max range of the image. The higher frequencies of the transform are dominated by noise, so it is found beneficial to use only the lower 16 of the resulting coefficients for classification.

\subsubsection{Pixel differences}

Pixel difference statistics at different scales are another way to capture the variation of textural energies. Basic average absolute difference between nearby pixels (horizontal and vertical offsets combined), is defined in Eq. 2, with pixel intensity at position $(i, j)$ denoted $I_{i, j}$ and the summation covering only those pixels that are within the segmentation mask $C$ of the cell.

$$
D(\delta)=\frac{1}{|C|} \sum_{i, j \in C}\left|I_{i+\delta, j}-I_{i, j}\right|+\left|I_{i, j+\delta}-I_{i, j}\right|
$$


When offset $\delta=1$, the difference is highest for fine speckled and homogeneous classes, whereas differences from 2 pixels apart $(\delta=2)$ are increased for coarse speckled and centromere. Repeated subsampling of the image by factor of 2 in each direction (following a suitable low-pass filter to avoid aliasing) and applying the pixel-difference operator creates a multi-scale textural signature. The first level of subsampling smooths out most of the finer textures, but brings the stronger gradients of centromere and nucleolar classes to pixel-level scale. Further levels of subsampling are not useful in this setting, as resulting images are too small to retain any relevant information.

The difference averages at the various scales are strongly linearly correlated with each other, but at characteristically different slopes for each class. We therefore derive the most classification benefit by taking pairwise ratios between measurements at different scales, and including them in the feature vector. The ratios are also free from dependency on overall brightness and contrast of the image - something which is difficult to achieve by explicit contrast normalisation.

\subsubsection{Morphology features}

Another way of comparing these textures, used by a number of contest participants $[15,11,12]$, is granulometry or morphological measurements of image slices at different thresholds. Similarly to [15], we consider 7 thresholds equally spaced between the extremes of intensity within each image, and compute 3 parameters from the connected objects produced at each threshold:

- mean area of each object relative to the area of the nucleus mask

- variance of all object relative areas

- average circularity of all the objects

Again following [15], we filter out objects below a certain size as noise. The resulting descriptor has $7 * 3=21$ features.

\subsubsection{Co-occurrence features}

Another well-established and common method of quantifying texture characteristics is the grey-level co-occurrence matrix (GLCM). It was used by a great many of the contestants as part of larger feature vectors, and so it is useful to compare its contribution. As the textures in question are isotropic, it is not necessary to consider different orientations separately, but offsets of different length can provide extra information about different scales of texture. We therefore include contrast, energy and correlation for matrices at $d=2$ and $d=4$ in the test vector.

\section{Results}

We report the predictions derived from each feature set in its corresponding section. For the contest protocol, the cell-level confusion tables are expressed as percentages relative to the total number of cells of each class within the 
test set. Additionally, the False Negative Rate for each class is given in the right-hand column. The sample-level confusion tables for the contest protocol are percentages relative to the number of samples of each class in the test set. For the leave-one-out protocol, additional breakdown of cell-level predictions within each sample is provided, both as number of cells and the percentages they represent within the particular sample.

Finally, the distribution overlap data is presented as distance maps, with dark points corresponding to closely overlapping distributions, and brighter ones being more separated. The samples are grouped by class and also by intensity, so that the first 3 samples are centromere and positive, the next 3 are centromere and intermediate, followed by the 5 homogeneous samples, similarly split by intensity, etc. This arrangement allows for easy visualisation of the expected performance of a feature set, based on the degree of block-diagonality within the distance map. For completeness, the full ordering of samples within the distance map is as follows: (Centromere:) 03, 13, 16, 07, 14, 19, (Homogeneous:) 01, 18, 22, 05, 21, (Nucleolar:) 08, 24, 04, 20, (Coarse-speckled:) 06, 12, 17, 10, 11, (Fine-speckled:) 09, 23, 02, 15, (Cytoplasmatic:) 26, 27, 25, 28.

A summary of the results, comparing the feature sets to each other, is given in Table 1.

\begin{tabular}{|l||c|c|c|c|}
\hline Evaluation & DCT & Pixel Diffs & Morphology & GLCM \\
\hline Training & $90.9 \%$ & $95.3 \%$ & $87.8 \%$ & $91.1 \%$ \\
Contest:cells & $52.3 \%$ & $56.5 \%$ & $52.2 \%$ & $35.3 \%$ \\
Contest:samples & $71.4 \%$ & $71.4 \%$ & $64.3 \%$ & $35.7 \%$ \\
Leave-1-out:cells & $53.5 \%$ & $53.7 \%$ & $50.6 \%$ & $39.4 \%$ \\
Leave-1-out:samples & $64.3 \%$ & $64.3 \%$ & $\mathbf{7 1 . 4 \%}$ & $60.7 \%$ \\
\hline
\end{tabular}

Table 1: Summary of accuracy rates for the different feature sets and forms of evaluation, highlighting overall best in bold type.

\subsection{DCT based descriptor}

For the contest protocol, the cross-validation accuracy on the training set is $90.9 \%$, while overall accuracy for the withheld test is $52.3 \%$. The full cell-level

\begin{tabular}{|l||r|r|r|r|r|r||r|}
\hline True Class & Centr & Homog & Nucl & Coarse & Fine & Cytopl & FNR \\
\hline Centr & 67.8 & 1.3 & 14.8 & 3.4 & 12.8 & 0.0 & 32.2 \\
Homog & 2.8 & 49.4 & 29.4 & 3.9 & 12.8 & 1.7 & 50.6 \\
Nucl & 20.9 & 25.9 & 20.9 & 12.9 & 18.7 & 0.7 & 79.1 \\
Coarse & 2.0 & 9.9 & 1.0 & 68.3 & 18.8 & 0.0 & 31.7 \\
Fine & 35.1 & 17.5 & 2.6 & 1.8 & 42.1 & 0.9 & 57.9 \\
Cytopl & 0.0 & 2.0 & 0.0 & 0.0 & 3.9 & 94.1 & 5.9 \\
\hline
\end{tabular}

Table 2: Cell-level confusion matrix for the test set using DCT features, as percentages of number of cells of true class in the test set 


\begin{tabular}{|l||r|r|r|r|r|r|}
\hline True Class & Centr & Homog & Nucl & Coarse & Fine & Cytopl \\
\hline Centromere & 100 & 0 & 0 & 0 & 0 & 0 \\
Homogen & 0 & 50 & 50 & 0 & 0 & 0 \\
Nucleolar & 50 & 0 & 50 & 0 & 0 & 0 \\
Coarse & 0 & 0 & 0 & 67 & 33 & 0 \\
Fine & 50 & 0 & 0 & 0 & 50 & 0 \\
Cytoplasm & 0 & 0 & 0 & 0 & 0 & 100 \\
\hline
\end{tabular}

Table 3: Sample-level confusion matrix for the test set using DCT features

\begin{tabular}{|l||r|r|r|r|r|r|}
\hline True Class & Centr & Homog & Nucl & Coarse & Fine & Cytopl \\
\hline Centromere & 100 & 0 & 0 & 0 & 0 & 0 \\
Homogen & 0 & 60 & 20 & 0 & 20 & 0 \\
Nucleolar & 25 & 50 & 25 & 0 & 0 & 0 \\
Coarse & 0 & 0 & 0 & 60 & 40 & 0 \\
Fine & 25 & 25 & 25 & 0 & 25 & 0 \\
Cytoplasm & 0 & 0 & 0 & 0 & 0 & 100 \\
\hline
\end{tabular}

Table 4: Sample-level confusion matrix for leave-one-out using DCT features

confusion matrix is given in Table 2. The sample-level accuracy for this protocol is $71.4 \%$, and the sample-level confusion is shown in Table 3 .

For the sample-based cross-validation, the sample-level accuracy is $64.3 \%$, and the cell-level is 53.5\%. Full confusion matrices are given in Tables 4 and 5 respectively. The numbers of cells predicted within each sample are listed in Table 18.

Finally, the distance matrix for the distributions of cells using this feature set are visualised in Fig. 2.

\subsection{Pixel differences}

For the contest protocol, the cross-validation accuracy on the training set is $95.3 \%$, while overall accuracy for the withheld test is $56.5 \%$. Full cell-level confusion matrix is given in Table 6 . The sample-level accuracy for this protocol is $71.4 \%$, and the sample-level confusion is shown in Table 7 .

\begin{tabular}{|l||r|r|r|r|r|r||r|}
\hline True Class & Centr & Homog & Nucl & Coarse & Fine & Cytopl & FNR \\
\hline Centromere & 78.7 & 0.8 & 5.3 & 5.0 & 10.1 & 0.0 & 21.3 \\
Homogen & 0.0 & 53.2 & 24.9 & 1.8 & 18.8 & 1.2 & 46.8 \\
Nucleolar & 21.6 & 46.5 & 16.6 & 8.3 & 7.1 & 0.0 & 83.4 \\
Coarse & 7.6 & 1.9 & 7.6 & 61.4 & 21.4 & 0.0 & 38.6 \\
Fine & 19.7 & 34.1 & 13.5 & 7.2 & 25.5 & 0.0 & 74.5 \\
Cytoplasm & 0.0 & 4.5 & 0.0 & 1.8 & 1.8 & 91.8 & 8.2 \\
\hline
\end{tabular}

Table 5: Cell-level confusion matrix for leave-one-out using DCT features, as percentages of number of cells of true class in the test set 


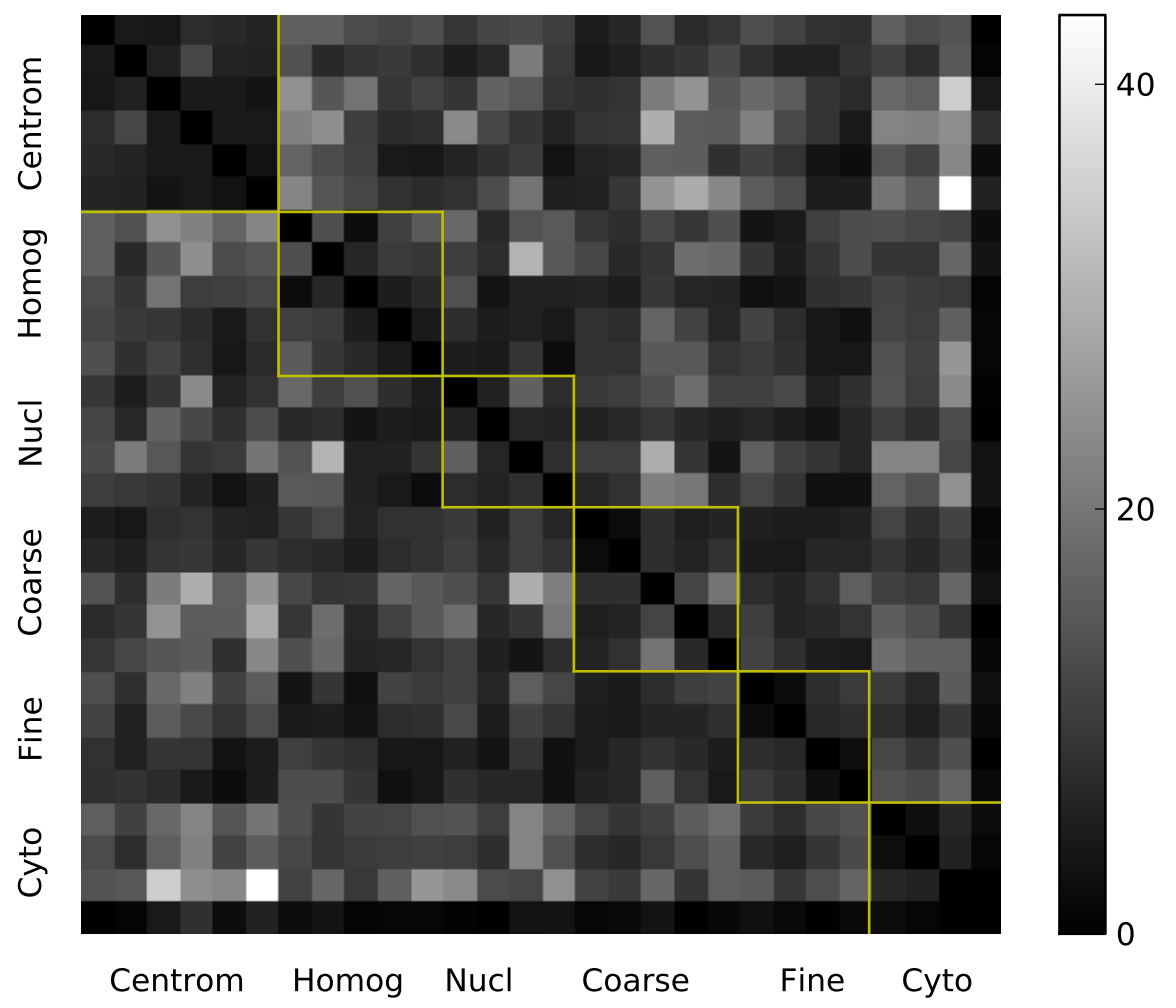

Figure 2: Distance map using DCT features

\begin{tabular}{|l||r|r|r|r|r|r||r|}
\hline True Class & Centr & Homog & Nucl & Coarse & Fine & Cytopl & FNR \\
\hline Centr & 83.2 & 2.0 & 12.1 & 1.3 & 1.3 & 0.0 & 16.8 \\
Homog & 15.6 & 48.9 & 10.6 & 3.9 & 18.3 & 2.8 & 51.1 \\
Nucl & 37.4 & 18.0 & 36.0 & 5.8 & 2.9 & 0.0 & 64.0 \\
Coarse & 0.0 & 12.9 & 1.0 & 61.4 & 21.8 & 3.0 & 38.6 \\
Fine & 39.5 & 16.7 & 0.9 & 0.0 & 43.0 & 0.0 & 57.0 \\
Cytopl & 13.7 & 0.0 & 0.0 & 0.0 & 3.9 & 82.4 & 17.6 \\
\hline
\end{tabular}

Table 6: Cell-level confusion matrix for the test set using pixel difference features, as percentages of number of cells of true class in the test set 


\begin{tabular}{|l||r|r|r|r|r|r|}
\hline True Class & Centr & Homog & Nucl & Coarse & Fine & Cytopl \\
\hline Centr & 100 & 0 & 0 & 0 & 0 & 0 \\
Homog & 50 & 50 & 0 & 0 & 0 & 0 \\
Nucl & 50 & 0 & 50 & 0 & 0 & 0 \\
Coarse & 0 & 0 & 0 & 67 & 33 & 0 \\
Fine & 50 & 0 & 0 & 0 & 50 & 0 \\
Cytopl & 0 & 0 & 0 & 0 & 0 & 100 \\
\hline
\end{tabular}

Table 7: Sample-level confusion matrix for the test set using pixel difference features

\begin{tabular}{|l||r|r|r|r|r|r|}
\hline True Class & Centr & Homog & Nucl & Coarse & Fine & Cytopl \\
\hline Centromere & 83 & 0 & 0 & 0 & 17 & 0 \\
Homogen & 0 & 60 & 20 & 0 & 20 & 0 \\
Nucleolar & 25 & 25 & 50 & 0 & 0 & 0 \\
Coarse & 0 & 0 & 0 & 80 & 20 & 0 \\
Fine & 50 & 0 & 0 & 0 & 50 & 0 \\
Cytoplasm & 0 & 0 & 0 & 0 & 0 & 100 \\
\hline
\end{tabular}

Table 8: Sample-level confusion matrix for leave-one-out using pixel difference features

For the sample-based cross-validation, the sample-level accuracy is $64.3 \%$, and the cell-level is 53.7\%. Full confusion matrices are given in Tables 8 and 9 respectively. The numbers of cells predicted within each sample are listed in Table 19.

Finally, the distance matrix for the distributions of cells using this feature set are visualised in Fig. 3 .

\subsection{Morphological features}

For the contest protocol, the cross-validation accuracy on the training set is $87.8 \%$, while overall accuracy for the withheld test is $52.2 \%$. Full cell-level confusion matrix is given in Table 10. The sample-level accuracy for this protocol is $64.3 \%$, and the sample-level confusion is shown in Table 11.

For the sample-based cross-validation, the sample-level accuracy is $71.4 \%$, and the cell-level is 50.6\%. Full confusion matrices are given in Tables 12 and

\begin{tabular}{|l||r|r|r|r|r|r||r|}
\hline True Class & Centr & Homog & Nucl & Coarse & Fine & Cytopl & FNR \\
\hline Centr & 70.6 & 0.8 & 7.0 & 6.2 & 14.3 & 1.1 & 29.4 \\
Homog & 1.5 & 53.0 & 16.4 & 4.2 & 23.0 & 1.8 & 47.0 \\
Nucl & 31.1 & 29.9 & 29.5 & 6.6 & 2.9 & 0.0 & 70.5 \\
Coarse & 8.6 & 1.9 & 8.6 & 64.3 & 16.2 & 0.5 & 35.7 \\
Fine & 28.8 & 27.4 & 1.4 & 8.7 & 33.2 & 0.5 & 66.8 \\
Cytopl & 5.5 & 8.2 & 0.0 & 5.5 & 8.2 & 72.7 & 27.3 \\
\hline
\end{tabular}

Table 9: Cell-level confusion matrix for leave-one-out using pixel difference features, as percentages of number of cells of true class in the test set 


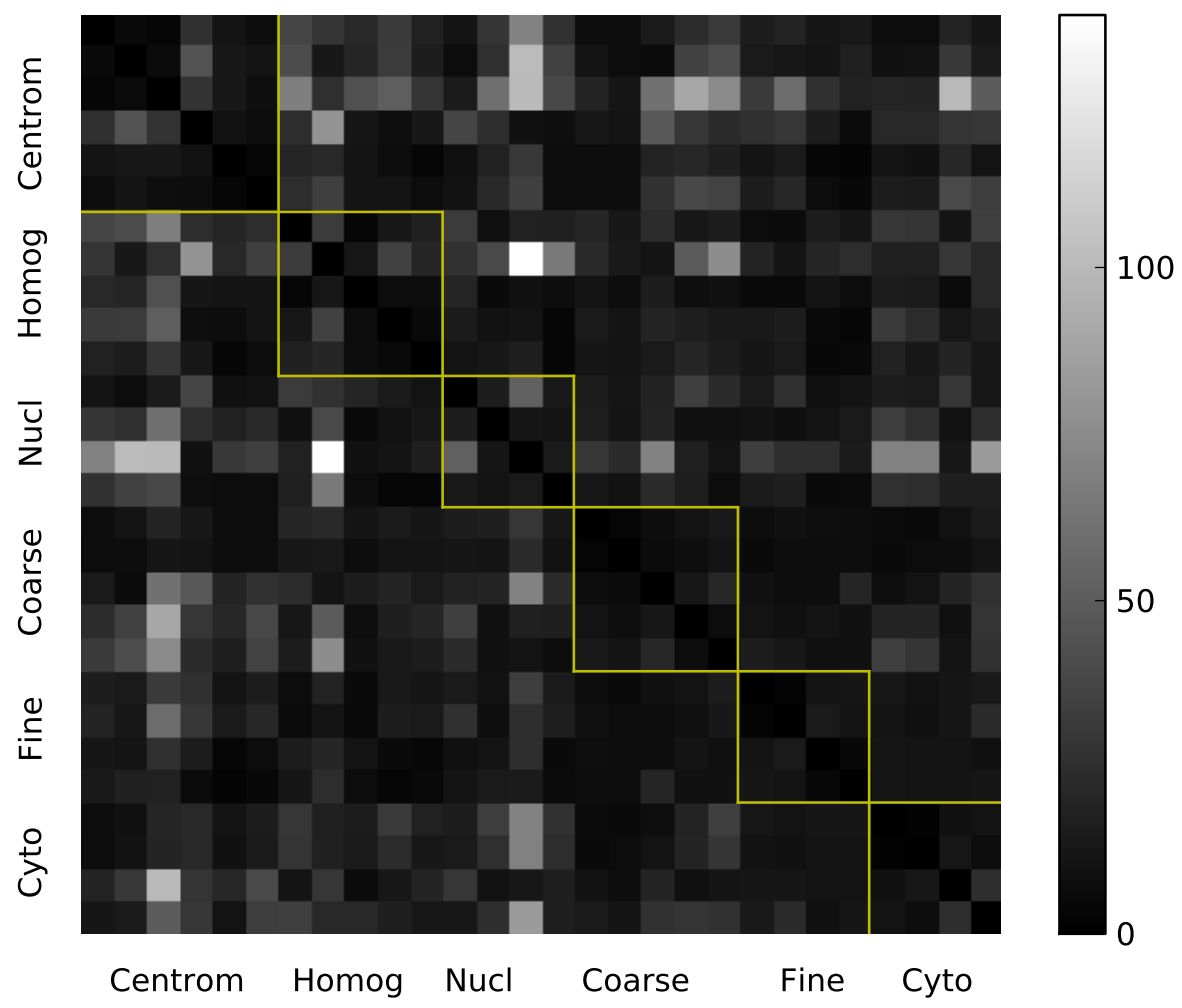

Figure 3: Distance map using pixel difference features

\begin{tabular}{|l||r|r|r|r|r|r||r|}
\hline True Class & Centr & Homog & Nucl & Coarse & Fine & Cytopl & FNR \\
\hline Centr & 59.7 & 0.0 & 18.1 & 21.5 & 0.0 & 0.7 & 40.3 \\
Homog & 16.7 & 40.0 & 1.7 & 6.1 & 33.3 & 2.2 & 60.0 \\
Nucl & 7.2 & 4.3 & 41.7 & 43.2 & 0.7 & 2.9 & 58.3 \\
Coarse & 1.0 & 10.9 & 1.0 & 70.3 & 14.9 & 2.0 & 29.7 \\
Fine & 21.1 & 29.8 & 1.8 & 3.5 & 43.9 & 0.0 & 56.1 \\
Cytopl & 3.9 & 0.0 & 7.8 & 3.9 & 0.0 & 84.3 & 15.7 \\
\hline
\end{tabular}

Table 10: Cell-level confusion matrix for the test set using morphological features, as percentages of number of cells of true class in the test set 


\begin{tabular}{|l||r|r|r|r|r|r|}
\hline True Class & Centr & Homog & Nucl & Coarse & Fine & Cytopl \\
\hline Centr & 67 & 0 & 0 & 33 & 0 & 0 \\
Homog & 50 & 50 & 0 & 0 & 0 & 0 \\
Nucl & 0 & 0 & 50 & 50 & 0 & 0 \\
Coarse & 0 & 0 & 0 & 67 & 33 & 0 \\
Fine & 0 & 50 & 0 & 0 & 50 & 0 \\
Cytopl & 0 & 0 & 0 & 0 & 0 & 100 \\
\hline
\end{tabular}

Table 11: Sample-level confusion matrix for the test set using morphological features

\begin{tabular}{|l||r|r|r|r|r|r|}
\hline True Class & Centr & Homog & Nucl & Coarse & Fine & Cytopl \\
\hline Centr & 83 & 0 & 0 & 17 & 0 & 0 \\
Homog & 20 & 60 & 0 & 0 & 20 & 0 \\
Nucl & 25 & 25 & 50 & 0 & 0 & 0 \\
Coarse & 0 & 0 & 0 & 80 & 20 & 0 \\
Fine & 0 & 50 & 0 & 0 & 50 & 0 \\
Cytopl & 0 & 0 & 0 & 0 & 0 & 100 \\
\hline
\end{tabular}

Table 12: Sample-level confusion matrix for leave-one-out using morphological features

13 respectively. The numbers of cells predicted within each sample are listed in Table 20.

Due to the threshold-based nature of this feature vector, none of the samples generate a full-rank co-variance (normality assumption does not hold), and so no distance matrix can be computed using this feature set.

\subsection{Co-occurrence features}

For the contest protocol, the cross-validation accuracy on the training set is $91.1 \%$, while overall accuracy for the withheld test is $35.3 \%$. Full cell-level confusion matrix is given in Table 14. The sample-level accuracy for this protocol is $35.7 \%$, and the sample-level confusion is shown in Table 15 .

For the sample-based cross-validation, the sample-level accuracy is $60.7 \%$, and the cell-level is $39.4 \%$. Full confusion matrices are given in Tables 16 and 17 respectively. The numbers of cells predicted within each sample are listed in Table 21.

\begin{tabular}{|l||r|r|r|r|r|r||r|}
\hline True Class & Centr & Homog & Nucl & Coarse & Fine & Cytopl & FNR \\
\hline Centr & 66.9 & 5.9 & 12.0 & 7.6 & 6.4 & 1.1 & 33.1 \\
Homog & 11.2 & 35.5 & 5.8 & 10.0 & 37.3 & 0.3 & 64.5 \\
Nucl & 37.8 & 16.6 & 35.3 & 5.0 & 4.1 & 1.2 & 64.7 \\
Coarse & 7.6 & 10.0 & 6.7 & 61.9 & 13.8 & 0.0 & 38.1 \\
Fine & 7.2 & 44.7 & 1.4 & 7.2 & 39.4 & 0.0 & 60.6 \\
Cytopl & 5.5 & 4.5 & 10.9 & 2.7 & 0.0 & 76.4 & 23.6 \\
\hline
\end{tabular}

Table 13: Cell-level confusion matrix for leave-one-out using morphological features, as percentages of number of cells of true class in the test set 


\begin{tabular}{|l||r|r|r|r|r|r||r|}
\hline True Class & Centr & Homog & Nucl & Coarse & Fine & Cytopl & FNR \\
\hline Centr & 75.8 & 0.7 & 7.4 & 2.7 & 2.0 & 11.4 & 24.2 \\
Homog & 16.1 & 23.3 & 16.1 & 18.3 & 23.3 & 2.8 & 76.7 \\
Nucl & 25.9 & 43.9 & 2.2 & 15.1 & 8.6 & 4.3 & 97.8 \\
Coarse & 0.0 & 11.9 & 1.0 & 38.6 & 24.8 & 23.8 & 61.4 \\
Fine & 23.7 & 37.7 & 8.8 & 0.0 & 29.8 & 0.0 & 70.2 \\
Cytopl & 19.6 & 0.0 & 15.7 & 9.8 & 0.0 & 54.9 & 45.1 \\
\hline
\end{tabular}

Table 14: Cell-level confusion matrix for the test set using co-occurrence features, as percentages of number of cells of true class in the test set

\begin{tabular}{|l||r|r|r|r|r|r|}
\hline True Class & Centr & Homog & Nucl & Coarse & Fine & Cytopl \\
\hline Centr & 67 & 0 & 0 & 0 & 0 & 33 \\
Homog & 0 & 50 & 50 & 0 & 0 & 0 \\
Nucl & 50 & 50 & 0 & 0 & 0 & 0 \\
Coarse & 0 & 0 & 0 & 33 & 33 & 33 \\
Fine & 50 & 50 & 0 & 0 & 0 & 0 \\
Cytopl & 0 & 0 & 0 & 0 & 50 & 50 \\
\hline
\end{tabular}

Table 15: Sample-level confusion matrix for the test set using co-occurrence features

\begin{tabular}{|l||r|r|r|r|r|r|}
\hline True Class & Centr & Homog & Nucl & Coarse & Fine & Cytopl \\
\hline Centr & 83 & 0 & 0 & 0 & 0 & 17 \\
Homog & 20 & 60 & 0 & 0 & 20 & 0 \\
Nucl & 25 & 75 & 0 & 0 & 0 & 0 \\
Coarse & 0 & 0 & 0 & 60 & 40 & 0 \\
Fine & 0 & 25 & 0 & 0 & 75 & 0 \\
Cytopl & 0 & 0 & 0 & 25 & 0 & 75 \\
\hline
\end{tabular}

Table 16: Sample-level confusion matrix for leave-one-out using co-occurrence features

\begin{tabular}{|l||r|r|r|r|r|r||r|}
\hline True Class & Centr & Homog & Nucl & Coarse & Fine & Cytopl & FNR \\
\hline Centr & 62.7 & 10.4 & 7.3 & 5.6 & 5.9 & 8.1 & 37.3 \\
Homog & 16.1 & 31.8 & 11.8 & 9.4 & 21.5 & 9.4 & 68.2 \\
Nucl & 22.4 & 58.5 & 2.9 & 7.9 & 6.2 & 2.1 & 97.1 \\
Coarse & 10.0 & 11.4 & 3.8 & 50.5 & 18.1 & 6.2 & 49.5 \\
Fine & 8.2 & 31.7 & 5.8 & 11.5 & 42.3 & 0.5 & 57.7 \\
Cytopl & 17.3 & 19.1 & 3.6 & 16.4 & 3.6 & 40.0 & 60.0 \\
\hline
\end{tabular}

Table 17: Cell-level confusion matrix for leave-one-out using co-occurrence features, as percentages of number of cells of true class in the test set 


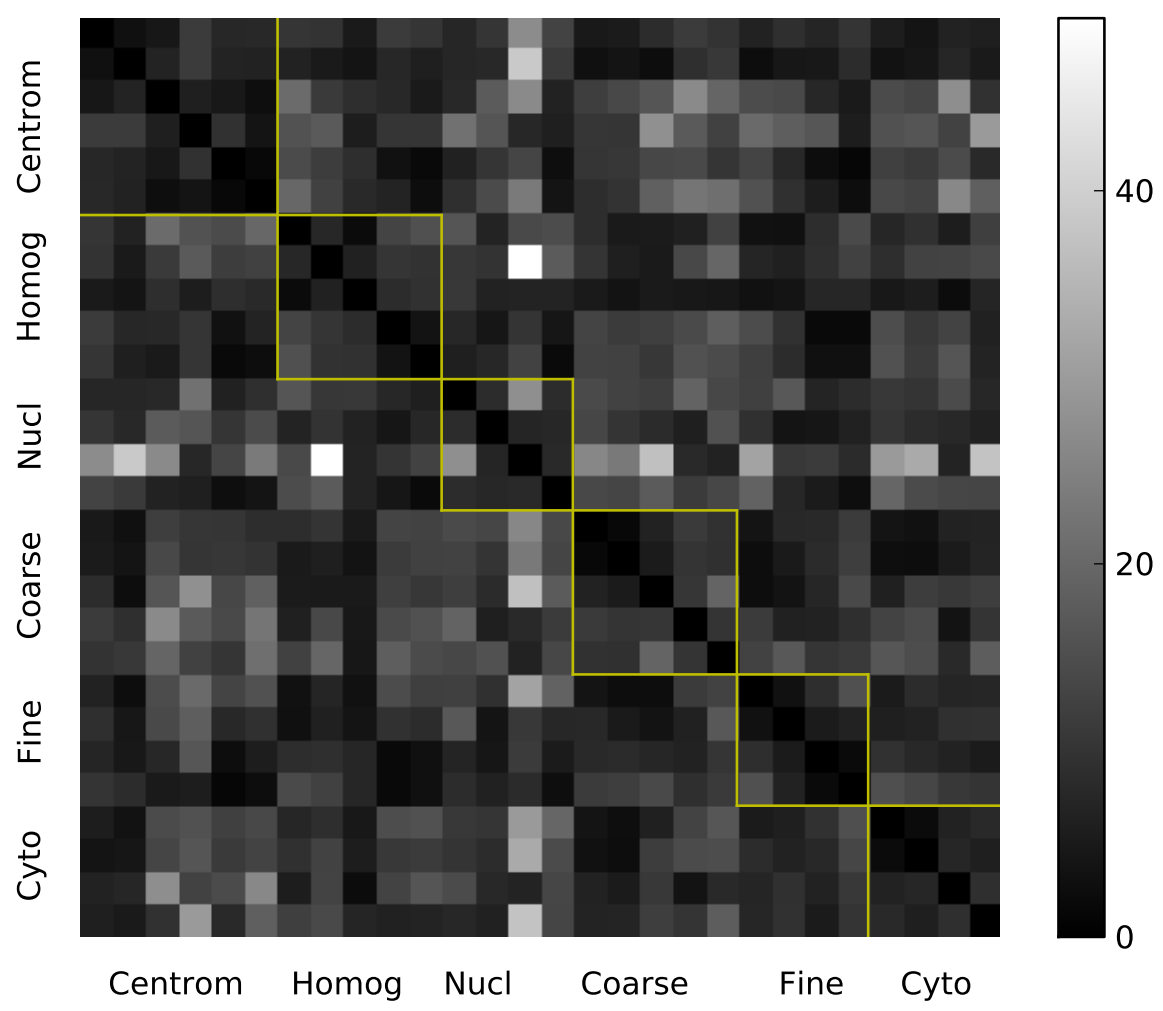

Figure 4: Distance map using co-occurrence features

Finally, the distance matrix for the distributions of cells using this feature set are visualised in Fig. 4.

\section{Discussion}

We have performed an experimental comparison of a number of different texture measures on a publicly available dataset of medical images. Our goal was not to achieve the best possible result, but to approach a better understanding of the intrinsic properties of this type of images and their class characteristics.

\subsection{Analysis of experimental results}

Of the feature sets tested, the morphological parameters clearly outperform the others on the ultimate measure of sample-level decisions in a leave-oneout protocol (see Table 1). An important point to note is that this difference 


\begin{tabular}{|c|c|c|c|c|c|c|c|c|c|c|c|c|}
\hline Image(Class) & \multicolumn{2}{|c|}{ Centromere } & \multicolumn{2}{|c|}{ Homogen } & \multicolumn{2}{|c|}{ Nucleolar } & \multicolumn{2}{|c|}{ Coarse } & \multicolumn{2}{|c|}{ Fine } & \multicolumn{2}{|c|}{ Cytoplasm } \\
\hline \#1 (Homog) & 0 & $0.0 \%$ & 58 & $95.1 \%$ & 0 & $0.0 \%$ & 0 & $0.0 \%$ & 3 & $4.9 \%$ & & $0.0 \%$ \\
\hline \#2 (Fine) & 5 & $10.4 \%$ & 1 & $2.1 \%$ & 22 & $45.8 \%$ & 7 & $14.6 \%$ & 13 & $27.1 \%$ & & $0.0 \%$ \\
\hline Centr) & 88 & $98.9 \%$ & 0 & $0.0 \%$ & 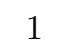 & $1.1 \%$ & 0 & $0.0 \%$ & 0 & $0.0 \%$ & & $0.0 \%$ \\
\hline $\mathrm{ucl}$ ) & 0 & $0.0 \%$ & 47 & $71.2 \%$ & 2 & $3.0 \%$ & 17 & $25.8 \%$ & 0 & $0.0 \%$ & & $0.0 \%$ \\
\hline Iomog) & 0 & $0.0 \%$ & 35 & $74.5 \%$ & 6 & $12.8 \%$ & 0 & $0.0 \%$ & 6 & $12.8 \%$ & & $0.0 \%$ \\
\hline \#6 ( & 12 & $17.6 \%$ & 0 & $0.0 \%$ & 0 & $0.0 \%$ & 55 & $80.9 \%$ & 1 & $1.5 \%$ & & $0.0 \%$ \\
\hline$\# 7$ & 50 & $89.3 \%$ & 0 & $0.0^{c}$ & 5 & $8.9 \%$ & 1 & $1.8 \%$ & 0 & $0.0 \%$ & 0 & $0.0 \%$ \\
\hline \#8 ( & 48 & $85.7 \%$ & 2 & $3.6 \%$ & 6 & $10.7 \%$ & 0 & $0.0 \%$ & 0 & $0.0 \%$ & 0 & $0.0 \%$ \\
\hline \#9 & 0 & $0.0 \%$ & 34 & $73.9 \%$ & 0 & $0.0 \%$ & 5 & $10.9 \%$ & 7 & $15.2 \%$ & 0 & $0.0 \%$ \\
\hline Coarse) & 1 & $3.0 \%$ & 0 & $0.0 \%$ & 1 & $3.0 \%$ & 23 & $69.7 \%$ & 8 & $24.2 \%$ & 0 & $0.0 \%$ \\
\hline \#11 & 2 & $4.9 \%$ & 1 & $2.4 \%$ & 14 & $34.1 \%$ & 3 & $7.3 \%$ & 21 & $51.2 \%$ & 0 & $0.0 \%$ \\
\hline \#12 & 0 & $0.0 \%$ & 1 & $2.0 \%$ & 0 & $0.0 \%$ & 46 & $93.9 \%$ & 2 & $4.1 \%$ & 0 & $0.0 \%$ \\
\hline \#13 (C & 25 & $54.3 \%$ & 0 & $0.0 \%$ & 0 & $0.0 \%$ & 14 & $30.4 \%$ & 7 & $15.2 \%$ & 0 & $0.0 \%$ \\
\hline \#14 (c & 34 & $54.0 \%$ & 3 & $4.8 \%$ & 1 & $1.6 \%$ & 0 & $0.0 \%$ & 25 & $39.7 \%$ & 0 & $0.0 \%$ \\
\hline \#15 (1 & 36 & $57.1 \%$ & 13 & $20.6 \%$ & 5 & $7.9 \%$ & & $1.6 \%$ & 8 & $12.7 \%$ & 0 & $0.0 \%$ \\
\hline \#16 (Centr) & 38 & $100.0 \%$ & 0 & $0.0 \%$ & 0 & $0.0 \%$ & 0 & $0.0 \%$ & 0 & $0.0 \%$ & 0 & $0.0 \%$ \\
\hline \#17 (Coar & 1 & $5.3 \%$ & 2 & $10.5 \%$ & 1 & $5.3 \%$ & 2 & $10.5 \%$ & 13 & $68.4 \%$ & 0 & $0.0 \%$ \\
\hline \#18 (Hon & 0 & $0.0 \%$ & 17 & $40.5 \%$ & 0 & $0.0 \%$ & 3 & $7.1 \%$ & 20 & $47.6 \%$ & 2 & $4.8 \%$ \\
\hline \#19 (Centr) & 46 & $70.8 \%$ & 0 & $0.0 \%$ & 12 & $18.5 \%$ & 3 & $4.6 \%$ & 4 & $6.2 \%$ & 0 & $0.0 \%$ \\
\hline \#20 (Nucl & 4 & $8.7 \%$ & 13 & $28.3 \%$ & 21 & $45.7 \%$ & 0 & $0.0 \%$ & 8 & $17.4 \%$ & 0 & $0.0 \%$ \\
\hline \#21 (Homog) & 0 & $0.0 \%$ & 0 & $0.0 \%$ & 59 & $96.7 \%$ & 0 & $0.0 \%$ & 2 & $3.3 \%$ & 0 & $0.0 \%$ \\
\hline \#22 (Homog) & 0 & $0.0 \%$ & 65 & $55.1 \%$ & 17 & $14.4 \%$ & 3 & $2.5 \%$ & 31 & $26.3 \%$ & 2 & $1.7 \%$ \\
\hline \#23 (Fine) & 0 & $0.0 \%$ & 23 & $45.1 \%$ & 1 & $2.0 \%$ & 2 & $3.9 \%$ & 25 & $49.0 \%$ & 0 & $0.0 \%$ \\
\hline \#24 ( & 0 & $0.0 \%$ & 50 & $68.5 \%$ & 11 & $15.1 \%$ & 3 & $4.1 \%$ & 9 & $12.3 \%$ & 0 & $0.0 \%$ \\
\hline \#25 (Cytopl) & 0 & $0.0 \%$ & 3 & $12.5 \%$ & 0 & $0.0 \%$ & 2 & $8.3 \%$ & 0 & $0.0 \%$ & 19 & $79.2 \%$ \\
\hline \#26 (C & 0 & $0.0 \%$ & 1 & 2. & 0 & $0.0 \%$ & 0 & $0.0 \%$ & 0 & $0.0 \%$ & 34 & $97.1 \%$ \\
\hline \#27 (Cyto & 0 & $0.0 \%$ & 0 & 0.0 & 0 & $0.0 \%$ & 0 & $0.0 \%$ & 2 & $5.3 \%$ & 36 & $94.7 \%$ \\
\hline \#28 (Cytopl) & 0 & $0.0 \%$ & 1 & $7.7 \%$ & 0 & $0.0 \%$ & 0 & $0.0 \%$ & 0 & $0.0 \%$ & 12 & $92.3^{\circ}$ \\
\hline
\end{tabular}

Table 18: Predictions within each sample using DCT features, as numbers of cells and percentages 


\begin{tabular}{|c|c|c|c|c|c|c|c|c|c|c|c|c|}
\hline Image(Class) & \multicolumn{2}{|c|}{ Centromere } & \multicolumn{2}{|c|}{ Homogen } & \multicolumn{2}{|c|}{ Nucleolar } & \multicolumn{2}{|c|}{ Coarse } & \multicolumn{2}{|c|}{ Fine } & \multicolumn{2}{|c|}{ Cytoplasm } \\
\hline \#1 (Homog) & 0 & $0.0 \%$ & 56 & $91.8 \%$ & 3 & $4.9 \%$ & 0 & $0.0 \%$ & 2 & $3.3 \%$ & & $0.0 \%$ \\
\hline \#2 (Fine) & 26 & $54.2 \%$ & 3 & $6.2 \%$ & 3 & $6.2 \%$ & 11 & $22.9 \%$ & 5 & $10.4 \%$ & & $0.0 \%$ \\
\hline \#3 (Centr) & 82 & $92.1 \%$ & 0 & $0.0 \%$ & 1 & $1.1 \%$ & 5 & $5.6 \%$ & 0 & $0.0 \%$ & & $1.1 \%$ \\
\hline \#4 (Nucl) & 21 & $31.8 \%$ & 25 & $37.9 \%$ & 6 & $9.1 \%$ & 14 & $21.2 \%$ & 0 & $0.0 \%$ & & $0.0 \%$ \\
\hline omog) & 0 & $0.0 \%$ & 29 & $61.7 \%$ & 8 & $17.0 \%$ & 0 & $0.0 \%$ & 10 & $21.3 \%$ & & $0.0 \%$ \\
\hline \#6 (Coars & 15 & $22.1 \%$ & 0 & $0.0 \%$ & 0 & $0.0 \%$ & 52 & $76.5 \%$ & 1 & $1.5 \%$ & & $0.0 \%$ \\
\hline \#7 (Centr) & 42 & $75.0 \%$ & 0 & $0.0 \%$ & 12 & $21.4 \%$ & 0 & $0.0 \%$ & 2 & $3.6 \%$ & & $0.0 \%$ \\
\hline \#8 (Nucl) & 48 & $85.7 \%$ & 7 & $12.5 \%$ & 1 & $1.8 \%$ & 0 & $0.0 \%$ & 0 & $0.0 \%$ & & $0.0 \%$ \\
\hline \#9 (Fine) & 0 & $0.0 \%$ & 11 & $23.9 \%$ & 0 & $0.0 \%$ & 7 & $15.2 \%$ & 28 & $60.9 \%$ & & $0.0 \%$ \\
\hline \#10 (Coarse) & 0 & $0.0 \%$ & 1 & $3.0 \%$ & 0 & $0.0 \%$ & 23 & $69.7 \%$ & 9 & $27.3 \%$ & & $0.0 \%$ \\
\hline \#11 (C & 0 & $0.0 \%$ & 0 & $0.0 \%$ & 16 & $39.0 \%$ & 17 & $41.5 \%$ & 8 & $19.5 \%$ & & $0.0 \%$ \\
\hline \#12 (Coarse) & 0 & $0.0 \%$ & 2 & $4.1 \%$ & 0 & $0.0 \%$ & 42 & $85.7 \%$ & 4 & $8.2 \%$ & & $2.0 \%$ \\
\hline \#13 (Centr) & 22 & $47.8 \%$ & 1 & $2.2 \%$ & 2 & $4.3 \%$ & 17 & $37.0 \%$ & 2 & $4.3 \%$ & 2 & $4.3 \%$ \\
\hline \#14 (Centr) & 10 & $15.9 \%$ & 2 & $3.2 \%$ & 4 & $6.3 \%$ & 0 & $0.0 \%$ & 46 & $73.0 \%$ & 1 & $1.6 \%$ \\
\hline \#15 (Fine) & 34 & $54.0 \%$ & 24 & $38.1 \%$ & 0 & $0.0 \%$ & 0 & $0.0 \%$ & 4 & $6.3 \%$ & 1 & $1.6 \%$ \\
\hline \#16 (Centr) & 38 & $100.0 \%$ & 0 & $0.0 \%$ & 0 & $0.0 \%$ & 0 & $0.0 \%$ & 0 & $0.0 \%$ & 0 & $0.0 \%$ \\
\hline \#17 (Coarse) & 3 & $15.8 \%$ & 1 & $5.3 \%$ & 2 & $10.5 \%$ & 1 & $5.3 \%$ & 12 & $63.2 \%$ & 0 & $0.0 \%$ \\
\hline \#18 (Homog) & 0 & $0.0 \%$ & 3 & $7.1 \%$ & 0 & $0.0 \%$ & 10 & $23.8 \%$ & 28 & $66.7 \%$ & 1 & $2.4 \%$ \\
\hline \#19 (Centr) & 58 & $89.2 \%$ & 0 & $0.0 \%$ & 6 & $9.2 \%$ & 0 & $0.0 \%$ & 1 & $1.5 \%$ & 0 & $0.0 \%$ \\
\hline \#20 (Nucl) & 6 & $13.0 \%$ & 10 & $21.7 \%$ & 28 & $60.9 \%$ & 0 & $0.0 \%$ & 2 & $4.3 \%$ & 0 & $0.0 \%$ \\
\hline \#21 (Homog) & 5 & $8.2 \%$ & 5 & $8.2 \%$ & 37 & $60.7 \%$ & 0 & $0.0 \%$ & 13 & $21.3 \%$ & 1 & $1.6 \%$ \\
\hline \#22 (Homog) & 0 & $0.0 \%$ & 82 & $68.9 \%$ & 6 & $5.0 \%$ & 4 & $3.4 \%$ & 23 & $19.3 \%$ & 4 & $3.4 \%$ \\
\hline \#23 (Fin & 0 & $0.0 \%$ & 19 & $37.3 \%$ & 0 & $0.0 \%$ & 0 & $0.0 \%$ & 32 & $62.7 \%$ & 0 & $0.0 \%$ \\
\hline \#24 (Nucl & 0 & $0.0 \%$ & 30 & $41.1 \%$ & 36 & $49.3 \%$ & 2 & $2.7 \%$ & 5 & $6.8 \%$ & 0 & $0.0 \%$ \\
\hline \#25 (Cytopl) & 0 & $0.0 \%$ & 6 & $25.0 \%$ & 0 & $0.0 \%$ & 6 & $25.0 \%$ & 6 & $25.0 \%$ & 6 & $25.0 \%$ \\
\hline \#26 (Cyto & 0 & $0.0 \%$ & 1 & 2. & 0 & $0.0 \%$ & 0 & $0.0 \%$ & 0 & $0.0 \%$ & 34 & $97.1 \%$ \\
\hline \#27 (Cyt & 2 & $3 \%$ & 0 & $0.0 \%$ & 0 & $0.0 \%$ & 0 & $0.0 \%$ & 0 & $0.0 \%$ & 36 & $94.7 \%$ \\
\hline \#28 (Cytopl) & 4 & $30.8 \%$ & 2 & $15.4 \%$ & 0 & $0.0 \%$ & 0 & $0.0 \%$ & 3 & $23.1 \%$ & 4 & $30.8 \%$ \\
\hline
\end{tabular}

Table 19: Predictions within each sample using pixel difference features, as numbers of cells and percentages 


\begin{tabular}{|c|c|c|c|c|c|c|c|c|c|c|c|c|}
\hline Image(Class) & \multicolumn{2}{|c|}{ Centromere } & \multicolumn{2}{|c|}{ Homogen } & \multicolumn{2}{|c|}{ Nucleolar } & \multicolumn{2}{|c|}{ Coarse } & \multicolumn{2}{|c|}{ Fine } & \multicolumn{2}{|c|}{ Cytoplasm } \\
\hline \#1 (Homog) & 0 & $0.0 \%$ & 36 & $59.0 \%$ & 3 & $4.9 \%$ & 0 & $0.0 \%$ & 22 & $36.1 \%$ & 0 & $0.0 \%$ \\
\hline \#2 (Fine) & 3 & $6.2 \%$ & 27 & $56.2 \%$ & 0 & $0.0 \%$ & 5 & $10.4 \%$ & 13 & $27.1 \%$ & 0 & $0.0 \%$ \\
\hline \#3 (Centr) & 78 & $87.6 \%$ & 0 & $0.0 \%$ & 7 & $7.9 \%$ & 0 & $0.0 \%$ & 0 & $0.0 \%$ & 4 & $4.5 \%$ \\
\hline \#4 (Nucl) & 6 & $9.1 \%$ & 34 & $51.5 \%$ & 12 & $18.2 \%$ & 6 & $9.1 \%$ & 8 & $12.1 \%$ & 0 & $0.0 \%$ \\
\hline \#5 (Homog) & 0 & $0.0 \%$ & 22 & $46.8 \%$ & 9 & $19.1 \%$ & 0 & $0.0 \%$ & 16 & $34.0 \%$ & 0 & $0.0 \%$ \\
\hline \#6 (Coarse) & 0 & $0.0 \%$ & 4 & $5.9 \%$ & 0 & $0.0 \%$ & 62 & $91.2 \%$ & 2 & $2.9 \%$ & 0 & $0.0 \%$ \\
\hline \#7 (Centr) & 35 & $62.5 \%$ & 0 & $0.0 \%$ & 15 & $26.8 \%$ & 1 & $1.8 \%$ & 5 & $8.9 \%$ & 0 & $0.0 \%$ \\
\hline \#8 (Nucl) & 54 & $96.4 \%$ & 0 & $0.0 \%$ & 0 & $0.0 \%$ & 0 & $0.0 \%$ & 0 & $0.0 \%$ & 2 & $3.6 \%$ \\
\hline \#9 (Fine) & 0 & $0.0 \%$ & 12 & $26.1 \%$ & 0 & $0.0 \%$ & 2 & $4.3 \%$ & 32 & $69.6 \%$ & 0 & $0.0 \%$ \\
\hline \#10 (Coarse) & 0 & $0.0 \%$ & 4 & $12.1 \%$ & 2 & $6.1 \%$ & 20 & $60.6 \%$ & 7 & $21.2 \%$ & 0 & $0.0 \%$ \\
\hline \#11 (Coarse) & 9 & $22.0 \%$ & 1 & $2.4 \%$ & 8 & $19.5 \%$ & 13 & $31.7 \%$ & 10 & $24.4 \%$ & 0 & $0.0 \%$ \\
\hline \#12 (Coarse) & 6 & $12.2 \%$ & 8 & $16.3 \%$ & 4 & $8.2 \%$ & 30 & $61.2 \%$ & 1 & $2.0 \%$ & 0 & $0.0 \%$ \\
\hline \#13 (Centr) & 35 & $76.1 \%$ & 1 & $2.2 \%$ & 5 & $10.9 \%$ & 5 & $10.9 \%$ & 0 & $0.0 \%$ & 0 & $0.0 \%$ \\
\hline \#14 (Centr) & 5 & $7.9 \%$ & 20 & $31.7 \%$ & 0 & $0.0 \%$ & 21 & $33.3 \%$ & 17 & $27.0 \%$ & 0 & $0.0 \%$ \\
\hline \#15 (Fine) & 12 & $19.0 \%$ & 36 & $57.1 \%$ & 3 & $4.8 \%$ & 5 & $7.9 \%$ & 7 & $11.1 \%$ & 0 & $0.0 \%$ \\
\hline \#16 (Centr) & 37 & $97.4 \%$ & 0 & $0.0 \%$ & 1 & $2.6 \%$ & 0 & $0.0 \%$ & 0 & $0.0 \%$ & 0 & $0.0 \%$ \\
\hline \#17 (Coarse) & 1 & $5.3 \%$ & 4 & $21.1 \%$ & 0 & $0.0 \%$ & 5 & $26.3 \%$ & 9 & $47.4 \%$ & 0 & $0.0 \%$ \\
\hline \#18 (Homog) & 0 & $0.0 \%$ & 8 & $19.0 \%$ & 0 & $0.0 \%$ & 13 & $31.0 \%$ & 20 & $47.6 \%$ & 1 & $2.4 \%$ \\
\hline \#19 (Centr) & 49 & $75.4 \%$ & 0 & $0.0 \%$ & 15 & $23.1 \%$ & 0 & $0.0 \%$ & 1 & $1.5 \%$ & 0 & $0.0 \%$ \\
\hline \#20 (Nucl) & 20 & $43.5 \%$ & 1 & $2.2 \%$ & 24 & $52.2 \%$ & 1 & $2.2 \%$ & 0 & $0.0 \%$ & 0 & $0.0 \%$ \\
\hline \#21 (Homog) & 37 & $60.7 \%$ & 2 & $3.3 \%$ & 1 & $1.6 \%$ & 0 & $0.0 \%$ & 21 & $34.4 \%$ & 0 & $0.0 \%$ \\
\hline \#22 (Homog) & 0 & $0.0 \%$ & 49 & $41.2 \%$ & 6 & $5.0 \%$ & 20 & $16.8 \%$ & 44 & $37.0 \%$ & 0 & $0.0 \%$ \\
\hline \#23 (Fine) & 0 & $0.0 \%$ & 18 & $35.3 \%$ & 0 & $0.0 \%$ & 3 & $5.9 \%$ & 30 & $58.8 \%$ & 0 & $0.0 \%$ \\
\hline \#24 (Nucl) & 11 & $15.1 \%$ & 5 & $6.8 \%$ & 49 & $67.1 \%$ & 5 & $6.8 \%$ & 2 & $2.7 \%$ & 1 & $1.4 \%$ \\
\hline \#25 (Cytopl) & 1 & $4.2 \%$ & 5 & $20.8 \%$ & 6 & $25.0 \%$ & 2 & $8.3 \%$ & 0 & $0.0 \%$ & 10 & $41.7 \%$ \\
\hline \#26 (Cytopl) & 3 & $8.6 \%$ & 0 & $0.0 \%$ & 4 & $11.4 \%$ & 0 & $0.0 \%$ & 0 & $0.0 \%$ & 28 & $80.0 \%$ \\
\hline \#27 (Cytopl) & 2 & $5.3 \%$ & 0 & $0.0 \%$ & 2 & $5.3 \%$ & 1 & $2.6 \%$ & 0 & $0.0 \%$ & 33 & $86.8 \%$ \\
\hline \#28 (Cytopl) & 0 & $0.0 \%$ & 0 & $0.0 \%$ & 0 & $0.0 \%$ & 0 & $0.0 \%$ & 0 & $0.0 \%$ & 13 & $100.0 \%$ \\
\hline
\end{tabular}

Table 20: Predictions within each sample using morphological features, as numbers of cells and percentages 


\begin{tabular}{|c|c|c|c|c|c|c|c|c|c|c|c|c|}
\hline Image(Class) & \multicolumn{2}{|c|}{ Centromere } & \multicolumn{2}{|c|}{ Homogen } & \multicolumn{2}{|c|}{ Nucleolar } & \multicolumn{2}{|c|}{ Coarse } & \multicolumn{2}{|c|}{ Fine } & \multicolumn{2}{|c|}{ Cytoplasm } \\
\hline \#1 (Homog) & 0 & $0.0 \%$ & 46 & $75.4 \%$ & 1 & $1.6 \%$ & 0 & $0.0 \%$ & 14 & $23.0 \%$ & & $0.0 \%$ \\
\hline \#2 (Fine) & 0 & $0.0 \%$ & 12 & $25.0 \%$ & 1 & $2.1 \%$ & 5 & $10.4 \%$ & 30 & 62.5 & & $0.0 \%$ \\
\hline \#3 (Centr) & 71 & $79.8 \%$ & 7 & $7.9 \%$ & 0 & $0.0 \%$ & 0 & $0.0 \%$ & 0 & $0.0 \%$ & 11 & $12.4 \%$ \\
\hline \#4 (1 & 20 & $30.3 \%$ & 21 & $31.8 \%$ & 5 & $7.6 \%$ & 19 & $28.8 \%$ & 0 & $0.0 \%$ & 1 & $1.5 \%$ \\
\hline omog) & 4 & $8.5 \%$ & 2 & $4.3 \%$ & 11 & $23.4 \%$ & 0 & $0.0 \%$ & 30 & $63.8 \%$ & & $0.0 \%$ \\
\hline \#6 ( ( & 8 & $11.8 \%$ & 0 & $0.0 \%$ & 0 & $0.0 \%$ & 58 & $85.3 \%$ & 0 & $0.0 \%$ & & $2.9 \%$ \\
\hline \#7 (C & 23 & $41.1 \%$ & 0 & $0.0 \%$ & 19 & $33.9 \%$ & 14 & $25.0 \%$ & 0 & $0.0 \%$ & & $0.0 \%$ \\
\hline \#8 (Nucl) & 28 & $50.0 \%$ & 26 & $46.4 \%$ & 1 & $1.8 \%$ & 0 & $0.0 \%$ & 0 & $0.0 \%$ & & $1.8 \%$ \\
\hline \#9 (Fine) & 0 & $0.0 \%$ & 27 & $58.7 \%$ & 0 & $0.0 \%$ & 17 & $37.0 \%$ & 1 & $2.2 \%$ & & $2.2 \%$ \\
\hline \#10 (Coarse) & 0 & $0.0 \%$ & 11 & $33.3 \%$ & 3 & $9.1 \%$ & 1 & $3.0 \%$ & 17 & $51.5 \%$ & & $3.0 \%$ \\
\hline \#11 (Coarse) & 13 & $31.7 \%$ & 3 & $7.3 \%$ & 5 & $12.2 \%$ & 14 & $34.1 \%$ & 0 & $0.0 \%$ & 6 & $14.6 \%$ \\
\hline \#12 (Coarse) & 0 & $0.0 \%$ & 9 & $18.4 \%$ & 0 & $0.0 \%$ & 33 & $67.3 \%$ & 3 & $6.1 \%$ & 4 & $8.2 \%$ \\
\hline \#13 (Centr) & 13 & $28.3 \%$ & 4 & $8.7 \%$ & 0 & $0.0 \%$ & 6 & $13.0 \%$ & 5 & $10.9 \%$ & 18 & $39.1 \%$ \\
\hline \#14 (Centr) & 21 & $33.3 \%$ & 21 & $33.3 \%$ & 6 & $9.5 \%$ & 0 & $0.0 \%$ & 15 & $23.8 \%$ & 0 & $0.0 \%$ \\
\hline \#15 (Fine) & 17 & $27.0 \%$ & 13 & $20.6 \%$ & 8 & $12.7 \%$ & 1 & $1.6 \%$ & 24 & $38.1 \%$ & 0 & $0.0 \%$ \\
\hline \#16 (Centr) & 37 & $97.4 \%$ & 0 & $0.0 \%$ & 1 & $2.6 \%$ & 0 & $0.0 \%$ & 0 & $0.0 \%$ & 0 & $0.0 \%$ \\
\hline \#17 (Coarse) & 0 & $0.0 \%$ & 1 & $5.3 \%$ & 0 & $0.0 \%$ & 0 & $0.0 \%$ & 18 & $94.7 \%$ & 0 & $0.0 \%$ \\
\hline \#18 (Homog) & 12 & $28.6 \%$ & 14 & $33.3 \%$ & 0 & $0.0 \%$ & 12 & $28.6 \%$ & 2 & $4.8 \%$ & 2 & $4.8 \%$ \\
\hline \#19 (Centr & 59 & $90.8 \%$ & 5 & $7.7 \%$ & 0 & $0.0 \%$ & 0 & $0.0 \%$ & 1 & $1.5 \%$ & 0 & $0.0 \%$ \\
\hline \#20 (Nucl) & 6 & $13.0 \%$ & 35 & $76.1 \%$ & 1 & $2.2 \%$ & 0 & $0.0 \%$ & 1 & $2.2 \%$ & 3 & $6.5 \%$ \\
\hline \#21 (Homog) & 34 & $55.7 \%$ & 0 & $0.0 \%$ & 26 & $42.6 \%$ & 0 & $0.0 \%$ & 1 & $1.6 \%$ & 0 & $0.0 \%$ \\
\hline \#22 (Homog) & 3 & $2.5 \%$ & 43 & $36.1 \%$ & 1 & $0.8 \%$ & 19 & $16.0 \%$ & 24 & $20.2 \%$ & 29 & $24.4 \%$ \\
\hline \#23 (Fin & 0 & $0.0 \%$ & 14 & $27.5 \%$ & 3 & $5.9 \%$ & 1 & $2.0 \%$ & 33 & $64.7 \%$ & 0 & $0.0 \%$ \\
\hline \#24 (Nucl & 0 & $0.0 \%$ & 59 & $80.8 \%$ & 0 & $0.0 \%$ & 0 & $0.0 \%$ & 14 & $19.2 \%$ & 0 & $0.0 \%$ \\
\hline \#25 (Cytopl) & 1 & $4.2 \%$ & 11 & $45.8 \%$ & 0 & $0.0 \%$ & 12 & $50.0 \%$ & 0 & $0.0 \%$ & 0 & $0.0 \%$ \\
\hline \#26 (Cytopl) & 6 & $17.1 \%$ & 7 & $20.0 \%$ & 1 & $2.9 \%$ & 1 & $2.9 \%$ & 3 & $8.6 \%$ & 17 & $48.6 \%$ \\
\hline \#27 (Cytc & 11 & $28.9 \%$ & 0 & $0.0 \%$ & 0 & $0.0 \%$ & 3 & $7.9 \%$ & 0 & $0.0 \%$ & 24 & $63.2 \%$ \\
\hline \#28 (Cytopl) & 1 & $7.7 \%$ & 3 & $23.1 \%$ & 3 & $23.1 \%$ & 2 & $15.4 \%$ & 1 & $7.7 \%$ & 3 & $23.1 \%$ \\
\hline
\end{tabular}

Table 21: Predictions within each sample using co-occurrence features, as numbers of cells and percentages 
of performance could not be predicted from the cell-level performance of the various classifiers, as the morphological features actually perform slightly worse for that evaluation, and its training cross-validation results are actually the worst of all the features tested. Although the performance difference seems clear, it is not possible to give an actual significance bracket for the results obtained, due to the limited size of the dataset and the nature of the prescribed evaluation protocols. It is also possible that the improved performance is simply due to the comparatively larger dimensionality of the morphological vector, rather than its intrinsically greater relevance to the class characteristics.

The DCT-based descriptor performs very poorly on nucleolar samples, but is perfect for cytoplasmatic ones. This is reflected in a strong block associated with the cytoplasmatic class in its distance matrix (bottom right in Fig. 2), as compared to a thin diagonal line for nucleolar.

GLCM is the worst performing feature set across the board, but is also the smallest vector. It particularly struggles with the nucleolar class, suggesting that, in its current formulation, it does not extend to large enough scales. Also, its quantisation level may not be optimal for this application.

Pixel-difference features seem most suited to the centromere class, but could also be used to separate the finer-grained classes (ie homogeneous and both speckled) from the rest.

Distance maps clearly visualise the fundamental problem: none of the features are able to produce a block-diagonal matrix which would indicate reliable similarity within classes and differentiation between them. There is too much variability within classes which is not adequately represented by the few examples that are available. Lack of class consistency within even just the positive samples of the homogeneous class is illustrated in every distance matrix: instead of a block, it is showing up as a diagonal cross, because the middle of the 3 available samples is very different from the other two. Similarly, many of the distance maps show the separation between positive and intermediate samples within each class, visible as two smaller blocks instead of one large block covering the whole class.

Careful inspection of the predicted class for each sample, as listed in Tables 18-21, also shows that some samples (for example \#16 and \#12) are predicted correctly with every feature set, while others (for example \#4 and \#17) are wrong in every case, suggesting that there may be an issue of variable image quality which is affecting the texture itself, however it is measured. Specific blurtolerant texture descriptors may need to be deployed to combat this problem.

\subsection{Further work}

The distribution distance matrix can be used as the basis of a nearestneighbour classifier, or an ensemble combining distance information from several feature vectors. Other ways of combining two or more different feature sets through either early or late fusion should also be explored, as there are indications of complementary information represented by different texture measures. As some feature types are more suited to identification of certain classes, they 
could also be combined in a cascade which filters out each class based on its most favourable features.

Another potentially fruitful approach to addressing the shortage of labelled image data is the use of semi-supervised methods. Leverage of large numbers of unlabelled HEp-2 images could allow development of much better understanding of the effect of imaging conditions on the resulting image texture, and compensating for these common variations in classification, for example through the use of manifold learning or subspace [16] methods.

\subsection{Conclusion}

We conclude strongly that cell-level performance of a classifier offers little guidance to its performance in whole-sample decisions, even in a simplistic majority-vote setting. This is supported by the recently published detailed report of the contest findings [5], which shows great variability between method rankings by cell-level and by sample-level performance. Consideration of the sample as a whole, including complete measurements from all the relevant cells, allows the application of a much richer set of pattern recognition methods, and is a better match for the ultimate goal of replicating the diagnostic decision of a physician. Whilst considerable progress is being made in identifying likely methods for single cell classification, we feel that assessment of their suitability for use in a realistic clinical system would require a larger quantity of data that more fully covers the variability of cell appearance.

\subsection{Acknowledgements}

This research was supported by Engineering and Physical Sciences Research Council.

[1] HEp-2 cells classification contest, 2012. URL: http://nerone.diiie.unisa.it/hep2contest/.

[2] P. Soda, Early experiences in the staining pattern classification of HEp-2 slides, in: CBMS, 2007.

[3] T.-Y. Hsieh, Y.-C. Huang, C.-W. Chung, Y.-L. Huang, HEp-2 cell classification in indirect immunofluorescence images, in: ICICS, 2009, pp. 1-4.

[4] P. Elbischger, S. Geerts, K. Sander, G. Ziervogel-Lukas, P. Sinah, Algorithmic framework for HEp-2 fluorescence pattern classification to aid auto-immune diseases diagnosis, in: ISBI '09, 2009, pp. 562-565.

[5] P. Foggia, G. Percannella, P. Soda, M. Vento, Benchmarking HEp-2 cells classification methods, Medical Imaging, IEEE Transactions on 32 (2013) 1878-1889.

[6] S. Di Cataldo, et al., Applying textural features to the classification of HEp-2 cell patterns in IIF images, in: ICPR, 2012. 
[7] I. Ersoy, et al., HEp-2 cell classification in IIF images using ShareBoost, in: ICPR, 2012.

[8] S. Ghosh, V. Chaudhary, Feature analysis for automatic classification of HEp-2 florescence patterns : Computer-aided diagnosis of auto-immune diseases, in: ICPR, 2012.

[9] K. Li, et al., Multiclass Boosting SVM Using Different Texture Features in HEp-2 Cell Staining Pattern Classification, in: ICPR, 2012.

[10] V. Snell, W. Christmas, J. Kittler, Texture and shape in fluorescence pattern identification for auto-immune disease diagnosis, in: ICPR, 2012.

[11] P. Strandmark, J. Ulén, F. Kahl, HEp-2 staining pattern classification, in: ICPR, 2012.

[12] G. Thibault, J. Angulo, Efficient statistical/morphological cell texture characterization and classification, in: ICPR, 2012.

[13] B. Wafa, et al., Classification of biological cells using bio-inspired descriptors, in: ICPR, 2012.

[14] MIVIA HEp-2 images dataset, http://mivia.unisa.it/datasets/biomedicalimage-datasets/hep2-image-dataset/, 2012.

[15] I. Theodorakopoulos, D. Kastaniotis, G. Economou, S. Fotopoulos, HEp-2 cells classification via fusion of morphological and textural features, in: BIBE, 2012.

[16] T.-K. Kim, J. Kittler, R. Cipolla, Discriminative learning and recognition of image set classes using canonical correlations, PAMI 29 (2007) 10051018.

Violet Snell received the MEng degree from the University of Oxford in 1996, and is now undertaking $\mathrm{PhD}$ studies in analysis of microscopic images at CVSSP, University of Surrey. Her earlier career covers research and software development for video processing applications.

William Christmas is currently Senior Research Fellow in Technology Transfer at CVSSP, University of Surrey. He studied at the University of Oxford and received his $\mathrm{PhD}$ degree from the University of Surrey. His research career covered a wide range of broadcast engineering projects at the $\mathrm{BBC}$, and real-time image processing and computer vision at BP Research International.

Josef Kittler received the B.A., Ph.D., and D.Sc. degrees from the University of Cambridge in 1971, 1974, and 1991, respectively. He founded the Centre for Vision, Speech and Signal Processing at the University of Surrey, UK. He teaches and conducts research in the subject area of machine intelligence, with a focus on biometrics, video and image database retrieval, automatic inspection, medical data analysis, and cognitive vision. 